\title{
Monocular deprivation in adult mice alters visual acuity and single-unit activity
}

\author{
Quentin S. Fischer, ${ }^{1,6}$ Aundrea Graves, ${ }^{2}$ Scott Evans, ${ }^{3}$ Marvin E. Lickey, ${ }^{2}$ \\ and Tony A. Pham ${ }^{1,4,5,6}$ \\ ${ }^{1}$ Department of Psychiatry and Behavioral Sciences, Baylor College of Medicine, Houston, Texas 77030, USA; ${ }^{2}$ Institute of \\ Neuroscience, University of Oregon, Eugene, Oregon 97403, USA; ${ }^{3}$ Department of Psychiatry and Behavioral Sciences, University \\ of Washington, School of Medicine, Seattle, Washington 98195, USA; ${ }^{4}$ Department of Molecular and Cellular Biology, Baylor \\ College of Medicine, Houston, Texas 77030, USA; ${ }^{5}$ Department of Neuroscience, Baylor College of Medicine, \\ Houston, Texas 77030, USA
}

It has been discovered recently that monocular deprivation in young adult mice induces ocular dominance plasticity (ODP). This contradicts the traditional belief that ODP is restricted to a juvenile critical period. However, questions remain. ODP of young adults has been observed only using methods that are indirectly related to vision, and the plasticity of young adults appears diminished in comparison with juveniles. Therefore, we asked whether the newly discovered adult ODP broadly reflects plasticity of visual cortical function and whether it persists into full maturity. Single-unit activity is the standard physiological marker of visual cortical function. Using a more optimized protocol for recording single-units, we find evidence of adult ODP of single-units and show that it is most pronounced in deep cortical layers. Furthermore, using visual evoked potentials (VEP), we find that ODP is equally robust in young adults and mature adults and is observable after just one day of monocular deprivation. Finally, we find that monocular deprivation in adults changes spatial frequency thresholds of the VEP, decreasing the acuity of the deprived pathway and improving the acuity of the non-deprived pathway. Thus, in mice, the primary visual cortex is capable of remarkable adaptation throughout life.

One of the best characterized models of developmental plasticity is the effect of monocular deprivation (MD) on ocular dominance in primary visual cortex (V1). MD causes a dramatic increase in responses of the non-deprived eye relative to the deprived eye. Previous studies, using single-unit recordings, have reported that ocular dominance plasticity (ODP) is restricted to a pre-pubertal critical period (Wiesel and Hubel 1963; LeVay et al. 1980; Fagiolini et al. 1994; Gordon and Stryker 1996; Issa et al. 1999). Recently, however, using different assays of cortical activity, ODP has been observed in young adults (Guire et al. 1999; Sawtell et al. 2003; Lickey et al. 2004; Pham et al. 2004; Tagawa et al. 2005). These new findings raise the exciting possibility that visual disorders thought treatable only in the very young are potentially treatable in adults. Therefore, it is important to understand the time course and underlying substrates of this adult plasticity.

In comparison with previous studies, these new findings may reflect three methodological advances. (1) Previous studies used single-unit recording, while recent studies used population assays of activity based on visual evoked potentials (VEP) or visually evoked gene expression. (2) Previous studies used barbiturate anesthesia (Wiesel and Hubel 1963; Hubel and Wiesel 1970), while recent studies used urethane anesthesia or no anesthesia (Sawtell et al. 2003; Lickey et al. 2004; Pham et al. 2004). (3) Previous studies examined changes in V1 contralateral to the deprived eye, while recent studies examined changes in both hemispheres.

Here we address three unresolved issues arising from observations of adult ODP. First, we asked whether adult ODP can be observed in single-unit responses. Previous single-unit recording

\footnotetext{
${ }^{6}$ Corresponding authors.

E-mail tapham@bcm.edu; fax (713) 798-8005.

E-mail qfischer@bcm.edu; fax (713) 798-8005.

Article is online at http://www.learnmem.org/cgi/doi/10.1101//m.392107.
}

studies have not found adult ODP but either lacked sufficient sampling and statistical power (Hubel and Wiesel 1970; Olson and Freeman 1980; Jones et al. 1984; Daw et al. 1992) or used barbiturate anesthesia, which can mask adult ODP (Pham et al. 2004). It is important to demonstrate plasticity of single-unit responses, because plasticity of VEP and visually evoked gene expression may reflect subthreshold changes that lack significance for visual cortical function. Accordingly, we investigated the plasticity of single-unit responses throughout the full cortical thickness under urethane anesthesia.

Second, we investigated whether the ODP observed in young adults continues into maturity or disappears with further maturation. Almost all of the data supporting adult ODP have come from mice and rats between two and four months of age, just beyond puberty (Guire et al. 1999; Sawtell et al. 2003; Lickey et al. 2004; Pham et al. 2004; Tagawa et al. 2005). Further, these young adults had less plasticity than juveniles. Here we track the potential for plasticity from juvenile life through mature adulthood.

Third, we examined whether it is likely that adult ODP reflects changes in visual perception. Previous VEP experiments measured responses to strong supra-threshold stimuli. Under these conditions, it is unclear whether plasticity of response amplitude is related to plasticity of perception. However, VEP stimulus detection thresholds are highly correlated with psychophysical visual acuity (Tyler et al. 1979; Allen et al. 1986). Therefore, we explored the plasticity of VEP detection thresholds for grating contrast and spatial frequency.

\section{Results}

Plastic change of single-unit activity in young adults under urethane anesthesia

We recorded receptive fields of isolated single-units in mice that were 55 to $110 \mathrm{~d}$ postnatal (P55-P110) on the day of recording. 
To avoid topographic sampling bias, we made three vertical penetrations spaced evenly across the mediolateral extent of binocular V1 (V1b) in each hemisphere (six penetrations/mouse). For each cell, the receptive field was plotted on a tangent screen using a hand-held projector. The preferred direction, orientation, size, and velocity of the stimulus were determined, and the optimal stimulus was then used to judge ocular dominance. Cells were scored for ocular dominance using the seven category scheme of Hubel and Wiesel (1962). In this scheme, a cell is given the score of 1 if it is driven exclusively by the contralateral eye, 4 if driven equally by both eyes, and 7 if driven exclusively by the ipsilateral eye (see Materials and Methods). To guard against subjective bias, the ocular dominance scores were assigned by an observer that was blind to the deprivation condition of the animal (non-deprived, right eye deprived, or left eye deprived).

In non-deprived young adult mice, the OD histograms of cells recorded from the left and right hemispheres were similar (Fig. 1A, left) and were contralaterally biased as described previously for juvenile mice (Gordon and Stryker 1996; Hensch et al. 1998; Fischer et al. 2004). Following MD, OD histograms were shifted to favor the non-deprived eye in both hemispheres (Fig. $1 \mathrm{~A}$, right). In the hemisphere contralateral to the deprived eye, histograms were shifted toward higher OD categories representing the non-deprived (ipsilateral) eye. In the hemisphere ipsilat-
A

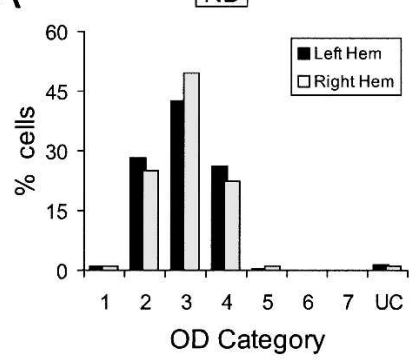

B

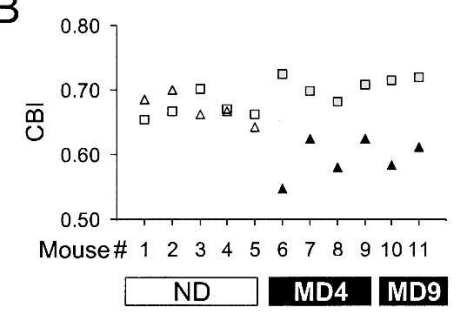

MD

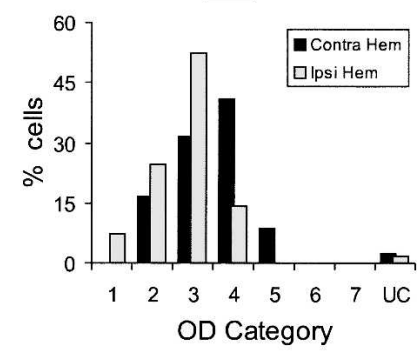

C

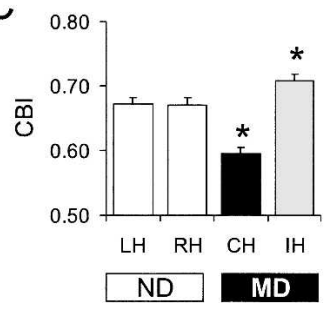

Figure 1. Ocular dominance plasticity can be demonstrated by singleunit recordings in urethane anesthetized young adult mice (P55-P110). (A) Ocular dominance histograms for each hemisphere of non-deprived mice (188 cells, five mice), and MD mice (213 cells, six mice). Category 1 cells were driven exclusively by the contralateral eye, category 7 cells were driven exclusively by the ipsilateral eye, and category 4 cells were driven equally by both eyes. UC, uncharacterized; ND, non-deprived; $\mathrm{MD}$, monocularly deprived (for 4 or $9 \mathrm{~d}$ ); Left Hem, left hemisphere; Right Hem, right hemisphere; Contra Hem, hemisphere contralateral to the deprived eye; Ipsi Hem, hemisphere ipsilateral to the deprived eye. (B) $\mathrm{CBI}$ scores calculated (see Materials and Methods) for each hemisphere in each mouse. Open triangles/squares represent the CBIs for the left/right hemisphere of individual non-deprived mice. Filled squares/triangles represent the CBIs for the hemisphere ipsilateral/contralateral to the deprived eye of individual MD mice. MD4, MD for four days; MD9, MD for nine days. (C) The average CBI for each hemisphere in non-deprived and $\mathrm{MD}$ mice. Asterisks $\left(^{*}\right)$ indicate a significant difference between the nondeprived hemispheres $(n=10)$ and the hemispheres contralateral $(n=6)$ or ipsilateral $(n=6)$ to the deprived eye $(P<0.005$, Bonferroni post-hoc tests). $\mathrm{LH}$, left hemisphere; $\mathrm{RH}$, right hemisphere; $\mathrm{CH}$, hemisphere contralateral to the deprived eye; $\mathrm{IH}$, hemisphere ipsilateral to the deprived eye. Error bars indicate SEM. eral to the deprived eye, histograms were shifted toward lower OD categories representing the non-deprived (contralateral) eye.

We quantified OD distributions by calculating a contralateral bias index (CBI, see Materials and Methods) for each hemisphere of each animal. A CBI score of 1 means that cells respond only to the eye contralateral to the recording site and a score of 0 means that cells respond only to the ipsilateral eye. In nondeprived mice, there was little difference between the CBIs of the right and heft hemispheres (open symbols, Fig. 1B), and therefore we pooled the right and left hemisphere data for statistical analysis. In MD mice, however, CBIs were consistently lower in the hemisphere contralateral to the deprived eye (black triangles) and consistently higher in the hemisphere ipsilateral to the deprived eye (gray squares, Fig. 1B). To test the significance of these results we compared the CBIs of the non-deprived hemispheres with the CBIs of the hemispheres contralateral or ipsilateral to the deprived eye (because in some cases we sutured the right eye and in others the left). These groups were significantly different $(P<0.0001$, one-way ANOVA), indicating an effect of MD. Pairwise comparisons further showed that, relative to the nondeprived hemispheres, CBIs of the hemispheres contralateral to the deprived eye were significantly lower $(P<0.0001$, Bonferroni post-hoc test) and CBIs of the hemispheres ipsilateral to the deprived eye were significantly higher $(P=0.0046$, Bonferroni posthoc test; Fig. 1C). Finally, we found no evidence that this effect of $\mathrm{MD}$ was increased by extending the period of deprivation beyond $4 \mathrm{~d}$ (Fig. 1B).

\section{Topography, sampling, and visual responses} were similar in non-deprived and MD mice

We were concerned that the hemispheric differences observed in MD mice might have arisen due to changes in retinotopy or to topographically biased sampling. To rule out these possibilities, we assessed retinotopic organization and sampling by constructing regression plots of receptive field azimuth versus mediolateral electrode position for each penetration in each hemisphere of non-deprived and MD mice. Comparable with previous studies (Drager 1975; Wagor et al. 1980; Gordon and Stryker 1996), binocular visual responses were obtained for penetrations within about the lateral $600 \mu \mathrm{m}$ of V1 and collectively represented the central $25^{\circ}$ of the contralateral visual field (see Materials and Methods). The regression plots consistently show linear relationships indicating similar topography in both hemispheres of nondeprived and MD mice (Fig. 2A). We found no difference between the left and right hemispheres of non-deprived mice or between the ipsilateral and contralateral hemispheres of MD mice; the slope and correlation coefficients were nearly identical. The regression plots also show that the topographic sampling of V1 and the visual field were similar in each hemisphere of nondeprived and MD mice, an important consideration because the binocularity of cells in V1 varies with azimuth. Moreover, for any given azimuth the OD shift toward the non-deprived eye was evident, reflected as an increase in CBI for the hemisphere ipsilateral to the deprived eye and a decrease in CBI for the hemisphere contralateral to the deprived eye (Fig. 2B). Finally, visual response strength and signal-to-noise ratio (see Materials and Methods) did not differ between non-deprived and MD mice $(P=0.98$ and 0.70, respectively, one-way ANOVA; Fig. 2C). Hence, the MD effect that we observed using single-unit recordings in young adults was not due to changes in retinotopy or neuronal responsiveness, or an artifact of topographically biased sampling.

\section{Adult ODP is most prominent in deep cortical layers}

Because some studies have reported that the effect of MD is greater in the extragranular layers of V1 (Shatz and Stryker 1978; 
ND

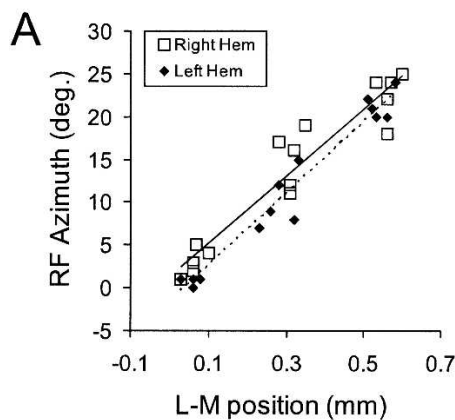

B

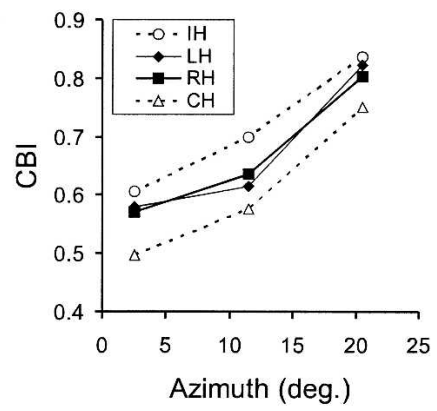

C

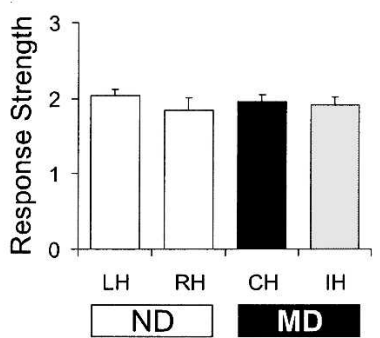

Figure 2. Retinotopy, topographic sampling, and visual responsiveness were similar in non-deprived and MD young adult mice (P55-P110). (A) Regression plots of receptive field azimuth vs. lateromedial electrode position were used to assess retinotopy and topographic sampling in nondeprived mice ( 30 penetrations, five mice) and MD mice ( 36 penetrations, six mice). The ordinate shows degrees of azimuth relative to the vertical meridian, while the abscissa indicates distance from the lateral edge of $\mathrm{V} 1 \mathrm{~b}$ (see Materials and Methods). (B) CBI plotted as a function of azimuth. Azimuth was binned in the following groups: $2.5^{\circ}$ midpoint (represents azimuth data from $-1^{\circ}$ to $7^{\circ}$ ), $11.5^{\circ}$ midpoint (represents azimuth data from $8^{\circ}$ to $16^{\circ}$ ), and $20.5^{\circ}$ midpoint (represents azimuth data from $17^{\circ}$ to $25^{\circ}$ ). Open symbols with dotted lines depict data in MD mice, while filled symbols and solid lines show data in non-deprived mice. $\mathrm{IH}$, hemisphere ipsilateral to the deprived eye; $\mathbf{L H}$, left hemisphere of non-deprived mice; $\mathrm{RH}$, right hemisphere of non-deprived mice; $\mathrm{CH}$, hemisphere contralateral to the deprived eye. (C) Average response strength and signal-to-noise ratio (see Materials and Methods) in each hemisphere of non-deprived and MD mice. Error bars indicate SEM.

Daw et al. 1992; Gordon and Stryker 1996), we sought to determine whether adult ODP varies by cortical layer. To examine this issue we divided our single-unit data into groups according to the depths below the cortical surface. A sample penetration, with two lesions (arrows) and their corresponding depths (in microns), is shown in Figure 3A. We marked one-third of the penetrations (the last of three) in each hemisphere, because an accurate measure of ODP using single-unit recordings requires sampling several mediolateral positions in close proximity. However, based on these reference lesions, we found that we were able to reliably determine the depths of recorded units (relative to the cortical surface) to within $\sim 50$ microns. Moreover, for each penetration in the same hemisphere, the last visually responsive cell was found at essentially the same depth. Finally, we have a high degree of confidence in our estimates of depth because we also routinely mark our VEP recording sites at a depth of 450 microns, and have found that the marks are consistently at the interface between layers 4 and 5. Therefore, we used the data from all of the penetrations in the subsequent analyses.

We divided our data into five groups. The first group $(<351$ $\mu \mathrm{m})$ likely consists primarily of layers $2 / 3$, while the second group (351-450 $\mu \mathrm{m}$ ) approximately encompasses layer 4 , and the three final groups $(451-550 \mu \mathrm{m}, 551-650 \mu \mathrm{m}$, and $>650 \mu \mathrm{m})$ are largely limited to layers $5 / 6$. We then determined CBIs based on units pooled from all cases. In MD mice, we found that the hemispheric difference in the CBIs was greatest in groups 3-5 (approximate layers 5/6), intermediate in group 1 (approximate layers 2/3), and least in group 2 (approximate layer 4) (Fig. 3B).

To test our impression that plasticity was greatest in the deeper layers, we combined the data for groups 1-2 (superficial layers) and 3-5 (deep layers) for each hemisphere in each animal ( $n=10$ non-deprived hemispheres, five hemispheres contralateral to the deprived eye and five hemispheres ipsilateral to the deprived eye) and performed a two-way ANOVA (Fig. 3C). The factors in the ANOVA were hemisphere (non-deprived, contralateral to deprived, and ipsilateral to deprived) and layer (super-
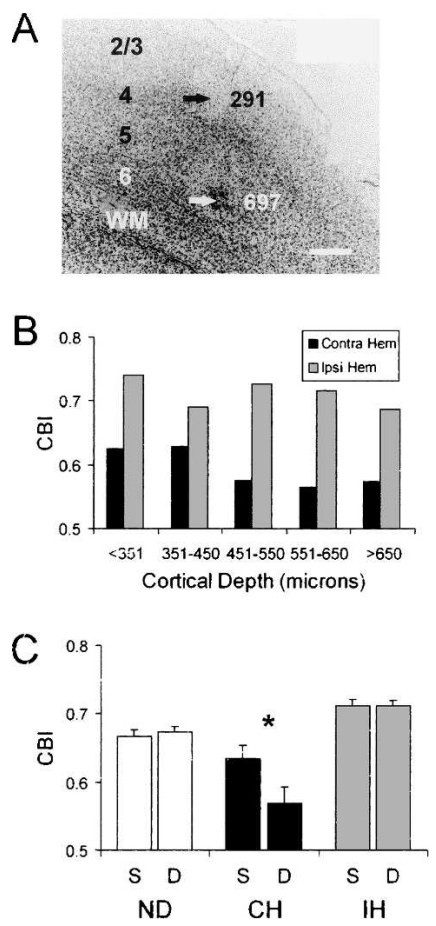

Figure 3. ODP is primarily expressed in the deep layers of $\mathrm{V} 1$ in young adult mice (P60-P106). (A) Micrograph showing electrolytic lesion sites for a penetration in $\mathrm{V} 1 \mathrm{~b}$. Arrows with numbers indicate lesion sites and their cortical depths (in microns). 2/3, layers $2 / 3 ; 4$, layer $4 ; 5$, layer $5 ; 6$, layer 6; WM, white matter. Scale bar $=200 \mu \mathrm{m}$. Dorsal is at the top and lateral is to the right of the figure. $(B)$ The average contralateral bias index ( $\mathrm{CBI}$, see Materials and Methods) of cells pooled for the indicated cortical depths (10-24 cells/bin) in five MD young adult mice (P60-P106). (C) The average $\mathrm{CBI}$ of cells pooled for cortical depths $\leq 450 \mu \mathrm{m}$ ( $\mathrm{S}$, superficial layers) or $>450 \mu \mathrm{m}$ (D, deep layers) in five non-deprived $(n=10$ hemispheres) and five MD mice ( $n=5$ hemispheres contralateral to the deprived eye, and 5 hemispheres ipsilateral to the deprived eye). The asterisk $\left(^{*}\right)$ denotes a significant difference between superficial and deep layers (hemisphere $\times$ layer interaction, $P<0.03$, two-factor ANOVA). Error bars indicate SEM. 


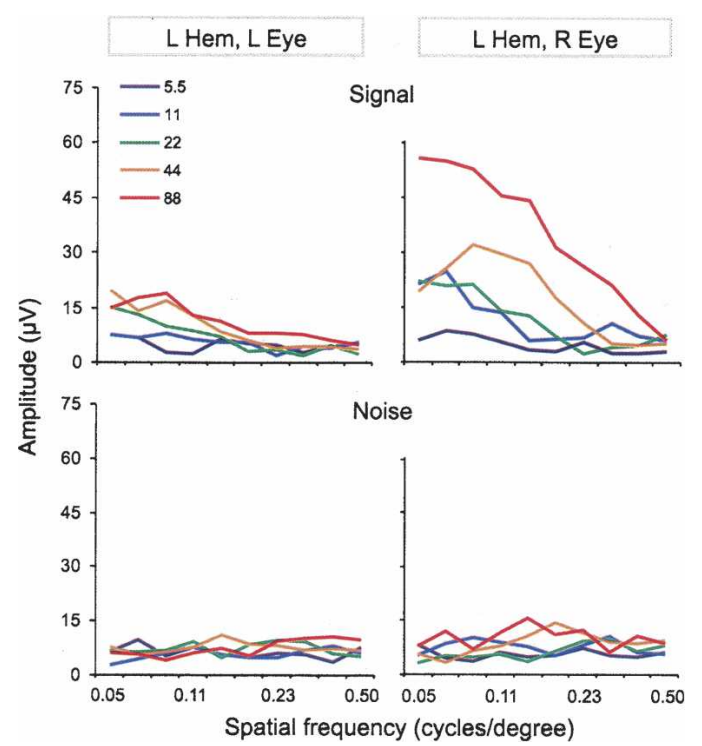

Figure 4. The VEP signal, but not noise, varied with both spatial frequency and contrast. Spatial frequency sweep data for the left hemisphere of a single non-deprived adult (P180) mouse. The VEP signal (top panels) and noise (bottom panels) are shown as a function of spatial frequency and grating contrast. The spatial frequencies (in cycles/degree) used were: $0.50,0.39,0.30,0.23,0.18,0.14,0.11,0.084,0.065,0.050$ (note: spatial frequency sweeps for this animal did not include stimuli at 0.65 cycles/degree). The visual stimuli were presented at five different contrasts (color-coded, see inset). Each curve is the average of 16 sweeps. L Hem, left hemisphere; L Eye, left eye; R Eye, right eye.

ficial or deep). We found a significant hemisphere $\times$ layer interaction $(P=0.03)$, indicating significantly greater plasticity in the deep layers compared to the superficial layers. Moreover, this effect was confined to the hemisphere contralateral to the deprived eye (Fig. 3C, black bars). These data show that representative sampling of deep cortical layers is necessary to observe robust adult ODP.

\section{Visual responses to varying contrast and spatial frequency determined using VEP}

To further explore the properties of adult plasticity, we used a swept VEP assay, which provides the capability of acquiring visual cortical responses quickly and efficiently (Tyler et al. 1979). This capability allows us to record from much older animals, even animals $>1$ yr of age, which are physiologically more fragile under anesthesia. Another advantage is that we can quantify visual responses to stimuli presented over a wide range of contrasts and spatial frequencies. To relate the effect of MD more directly to visual acuity we recorded VEPs in response to stimuli that bracketed the detection threshold, varying both contrast and spatial frequency. This protocol generated data that allowed construction of curves showing the amplitude of response as a function of both the contrast and spatial frequency.

As in previous studies (Lickey et al. 2004; Pham et al. 2004), the VEP ampli- tude was defined as the sum of frequency components in the electrocorticogram that were synchronized with the reversal grating (see Materials and Methods). A sample result from one animal is shown in Figure 4. The VEP amplitude decreased with increasing spatial frequency over the range of 0.05 to 0.5 cycles/degree and increased over the range of $5 \%$ to $88 \%$ grating contrast (Fig. 4 , top). Stimulation of the eye contralateral to the recording site (crossed pathway) produced larger amplitudes than stimulation of the ipsilateral eye (the uncrossed pathway). To ensure that the differences in VEP amplitude were stimulus related, we also assessed the amplitude of electrocorticogram frequencies that were not synchronized with the reversal grating (denoted hereafter as "noise"; see Materials and Methods). We found no relationship between noise and spatial frequency (Fig. 4, bottom). There was, however, a very weak relationship between noise and stimulus contrast; the electrocorticogram tended to be slightly more active at higher stimulus contrasts. Note in Figure 4 that at $88 \%$ contrast the signal amplitude in the left cortex from the right eye falls to the noise level at $\sim 0.5$ cycles/degree. This spatial frequency is about equal to behaviorally determined binocular spatial frequency thresholds in mice (Prusky et al. 2000; Wong and Brown 2006).

\section{Mature adults possess significant ODP}

Because there is a lack of data for mature adults, we collected data from mice between 180 and $390 \mathrm{~d}$ of age, essentially middle-age in this species. To represent data from these mature non-deprived and MD adults, we averaged normalized VEP amplitudes from all animals in each group (see Materials and Methods). The group curves consistently showed greater amplitudes at higher contrasts and lower spatial frequencies (Fig. 5), consistent with human data (Tyler et al. 1979; Allen et al. 1986). In non-deprived mice (Fig. 5A), the responses of the right and left hemispheres were symmetric; in both hemispheres the crossed pathway produced greater signal than the uncrossed pathway. In mice subjected to right eye MD for four days (Fig. 5B), this symmetry was

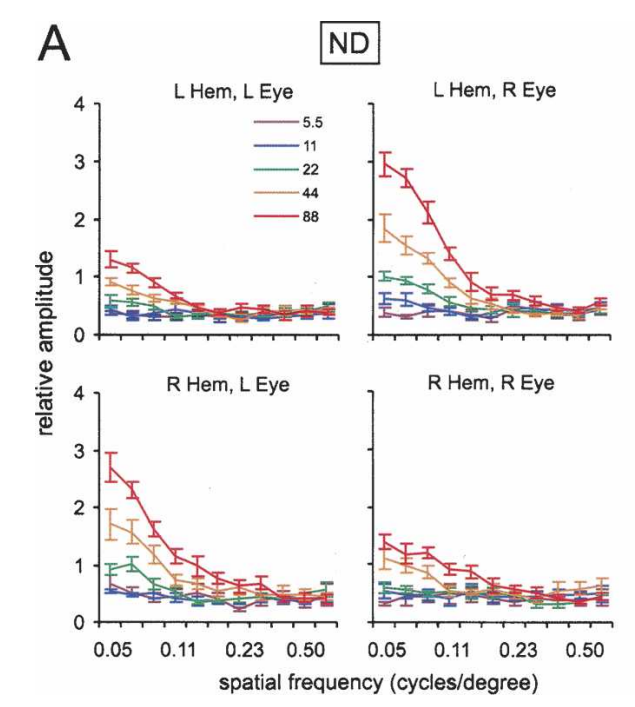

Figure 5. MD strongly alters swept spatial frequency curves in mature adult mice (P181-P390). $(A, B)$ Averaged normalized VEP amplitudes are shown as a function of spatial frequency and contrast for non-deprived $(A)$ and four-day right eye MD $(B)$ mature adult mice. VEPs were elicited by using a contrast grating stimulus that swept from 0.65 to 0.05 cycles/degree. Five different contrast levels were used, $5.5 \%, 11 \%, 22 \%, 44 \%$, and $88 \%$ (color-coded, see insets). The right and left hemispheres were recorded simultaneously while either the right or left eye was stimulated; results for the crossed and uncrossed pathways of each eye are shown. The ordinate shows the amplitude normalized to a scaling factor for the hemisphere (see Materials and Methods). Each curve represents the average of 10 animals. Error bars indicate SEM. 
lost; in the left hemisphere, responses in the crossed and uncrossed pathways were almost equal, but in the right hemisphere the dominance of the crossed pathway was greater than normal. This means that the non-deprived (left) eye evoked greater responses than the deprived (right) eye, a clear effect of MD in mature adult mice. This effect was observed most clearly in the uncrossed pathway; where compared to normal mice, the right eye response was much weaker and the left eye response was much stronger.

We quantified the ocular dominance shift by calculating an ocular dominance index (ODI) that compared the strength of crossed and uncrossed responses in each hemisphere (see Materials and Methods). The ODI has a range of $0-1.0$, with 0.5 signifying equal responses to stimulation of either eye. Values $>0.5$ indicate dominance by the contralateral eye and values $<0.5$ indicate dominance by the ipsilateral eye.

To understand how ODP changes with maturation, we recorded from six groups of mice, non-deprived and MD juveniles (P26-P36), non-deprived and MD young adults (P90-P180), nondeprived and MD mature adults (P181-390). Scatter plots of individual ODI scores from both hemispheres (Fig. 6A) show that in non-deprived mice ODIs of the left and right hemispheres were approximately equal. In MD mice of all ages, an MD effect was indicated by lower ODIs in the left hemisphere than in the right. For statistics, we averaged the ODIs by age groups (Fig. 6B) and analyzed significance using three-way ANOVA. The effect of MD was confirmed by a deprivation $\times$ hemisphere interaction $(P<0.0005)$, indicating a hemispheric difference in the MD groups but not in the non-deprived groups. This hemispheric difference may be greater in juveniles than in mature adults; however, we did not find statistical support for this conclusion since the deprivation $\times$ hemisphere $\times$ age interaction was not significant $(P=0.69)$. There is a tendency for the ODIs in the mature adult animals to be higher than in the juveniles, especially in the left hemisphere (effect of age, $P<0.01$; age $\times$ hemisphere interaction, $P<0.001$ ). To test whether the MD effect occurred in the right or left hemisphere, we performed post-hoc group comparisons using Bonferroni correction. Both the left and right hemispheres of MD juvenile and mature adult mice differed from the left and right hemispheres of nondeprived mice $(P<0.01$ in all cases). In summary, monocular deprivation caused a shift of ocular dominance in favor of the non-deprived eye; this shift occurred in both hemispheres and, based on statistical analysis, did not appear to decline significantly with age.

\section{Adult ocular dominance plasticity demonstrated using swept VEPs has a rapid onset}

We also investigated the time course of adult ocular dominance plasticity, its onset, and saturation, by varying the duration of MD. Figure 7 shows ODI scores for each hemisphere of the adult mice (P82-P232), comparing non-deprived mice with mice subjected to MD for one day (MD1), two days (MD2), four days (MD4), or nine days (MD9). Note that following MD the MD1 group was exposed to $24 \mathrm{~h}$ of continuous light, whereas the other groups were kept on a standard 12-h light/12-h dark cycle. The data for the non-deprived and MD4 groups are the same as in Figure 6. A two-way ANOVA comparing MD1, MD2, and MD9 groups shows a significant effect of hemisphere $(P<0.0005)$, but no effect of MD group or MD group $\times$ hemisphere interaction. The hemisphere effect indicates that there was a significant effect of $\mathrm{MD}$; the absence of a hemisphere $\times$ deprivation interaction indicates that the plasticity was not different among the MD1, MD2, or MD9 groups. A single pairwise comparison also shows that the hemispheric difference for MD1 was significant
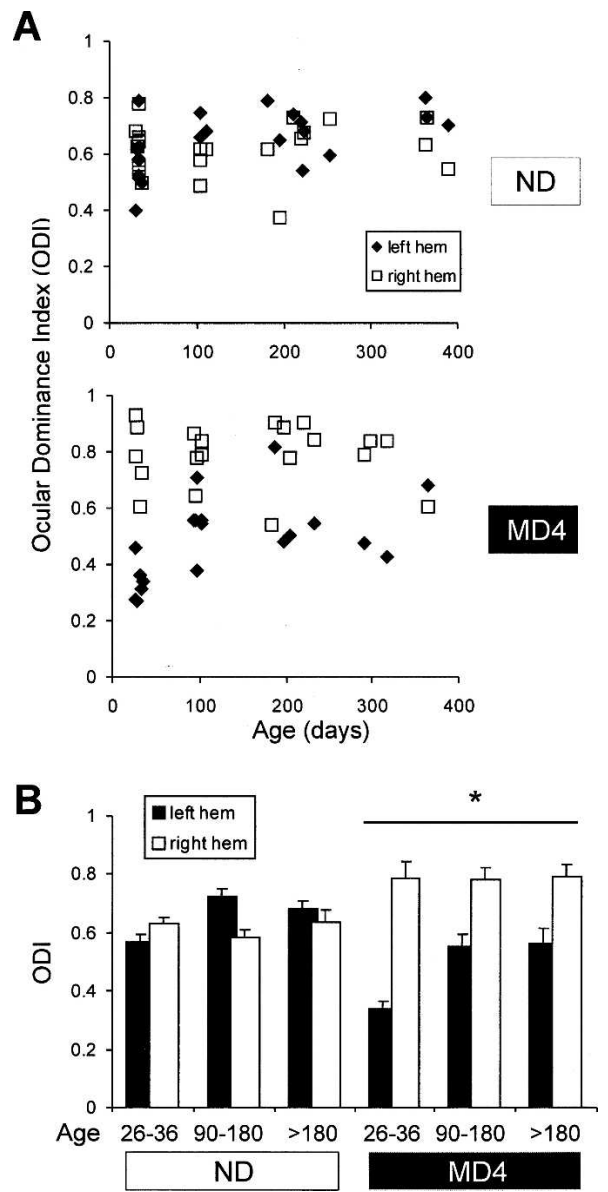

Figure 6. Ocular dominance plasticity is equally robust in young adult (P90-180) and mature adult (>P180) mice. We represented the ocular dominance of each hemisphere in each mouse using an ocular dominance index (ODI, see Materials and Methods). ( $A$ ) ODIs (ordinate) for the left (filled symbols) and right (open symbols) hemispheres of nondeprived mice (top) or four-day right eye MD mice (bottom) as a function of age at the time of recording (abscissa). ODls were calculated using data obtained at $88 \%$ contrast and a spatial frequency of 0.05 cycles/ degree. Each data point represents a single hemisphere. (B) The average ODI for each hemisphere of non-deprived and MD mice for juveniles (P26-P36, five mice), young adults (P90-P180, five mice), and mature adults (>P180, 10 mice). The asterisk and bar indicate that there was an overall hemispheric difference in the MD groups (hemisphere $\times$ deprivation interaction, $P<0.005$, three-way ANOVA). Error bars indicate SEM.

( $P=0.04$, Student's $t$-test). These data indicate that adult ODP is observable after as little as one day of MD.

\section{MD alters the spatial frequency threshold}

Thus far we have described how MD affects responses to stimuli well above the perceptual threshold. In order to understand the effect of MD on visual acuity, it is important to examine the effect near the threshold of visual detection. VEP thresholds have been shown to be closely related to psychophysical thresholds (Tyler et al. 1979; Allen et al. 1986). Accordingly, we devised an algorithm to define a detection threshold based on swept VEP data. We set a criterion for the minimal amplitude of a detectable response and defined the spatial frequency threshold as the highest spatial frequency that produced responses above the criterion. The criterion was equal to the average noise from all 11 bins (see Fig. 4) plus one standard deviation of the noise (see Materials and Methods). This is a fairly stringent criterion. 


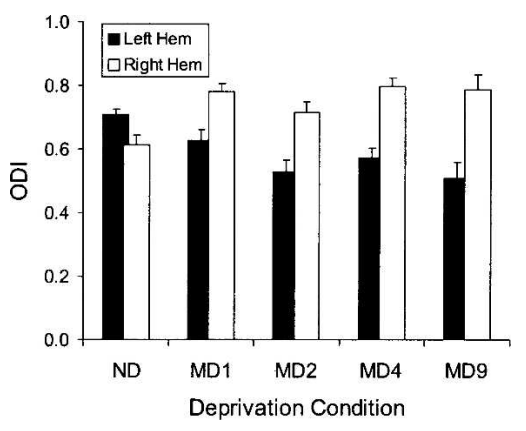

Figure 7. Adult ODP demonstrated by VEP has a rapid onset. The average ODI is shown for each hemisphere of mice which were nondeprived (ND, 13 mice) or right eye deprived for: one day (MD1, four mice), two days (MD2, six mice), four days (MD4, 12 mice), or nine days (MD9, three mice). All mice were maintained on a 12-h light/12-h dark cycle, except MD1 mice which were housed in continuous light for $24 \mathrm{~h}$. Error bars indicate SEM.

We averaged data from all young adult and mature adult animals (P90-P390), yielding the spatial frequency threshold functions shown in Figure 8A. As expected, the threshold value ( $Y$-axis) increased with increasing contrast of the stimulus $(X$ axis). In the crossed (contralateral) pathways, we found no effect of MD. The curves for the right and left eyes are nearly superimposed in both the non-deprived and MD groups. In the uncrossed pathways, however, there was a clear effect of MD. In non-deprived animals (ND), the spatial frequency threshold for the right uncrossed pathway seemed to be slightly higher than the left. In contrast, in the MD4 group, the left uncrossed pathway was clearly superior to the right. This interocular difference appears to reflect both a gain in acuity for the non-deprived (left) eye and a loss in acuity for the deprived (right) eye.

For statistical analysis we expressed the thresholds of each pathway as an acuity index, defined as the mean of the three thresholds obtained at 22\%, 44\%, and $88 \%$ contrast (Fig. 8B). We used a general linear model and three-factor ANOVA to test for significance (factors: eye, pathway, deprivation). This analysis showed a significant effect of MD on acuity indexes, as indicated by the deprivation $\times$ eye interaction $(P=0.001)$. The deprivation $\times$ eye interaction means that the deprived eye was different from the non-deprived eye. Also, there was a significant effect of pathway; the crossed pathway had a significantly higher acuity index than the uncrossed $(P<0.001)$. This simply reflects the strong contralateral eye dominance in the mouse visual system.

The effect of MD appeared to be much larger in the uncrossed pathways than in the crossed pathways (Fig. 8A,B), but our analysis suggested only a trend level interaction (eye $\times$ pathway $\times$ deprivation interaction, $P=0.2$ ). To explore this issue further, we performed pairwise comparisons between the non-deprived group and the MD group for each of the four pathways. There was a significant difference between the nondeprived and MD groups for the uncrossed pathway of both eyes ( $P=0.03$; Bonferroni post-hoc tests), but the effect in the crossed pathways was not significant $(P>0.8$, Bonferroni post-hoc tests; Fig. 8B). We conclude that, in mice older than P90, MD changes visual thresholds, improving the acuity of the non-deprived eye and decreasing the acuity of the deprived eye. Moreover, this effect was most apparent in the uncrossed pathways.

\section{Discussion}

The adult brain clearly has the potential for functional adaptation. This potential underlies the recovery from brain injury and disease as well as normal adult learning. However, the extent of adult plasticity and its limitations are not fully known. The effect of MD in V1, once thought limited to juveniles, has recently been shown to continue into young adulthood. Yet, questions regarding the scope, extent, and generality of adult ODP have remained. Here, we report that the effects of MD can be measured as a change in a variety of functionally relevant visual parameters, including activity of single-units and spatial frequency thresholds (an index of acuity). This plasticity has a very rapid onset and is robust even in adults near middle-age.

\section{Adult ODP revealed by single-unit recordings}

We found that MD of $4 \mathrm{~d}$ or more produced changes of ocular dominance in single-units recorded under urethane anesthesia in mice that were $60-106 \mathrm{~d}$ old. In six out of six cases, the CBI of the hemisphere contralateral to the MD was lower than the CBI of the hemisphere ipsilateral to the MD. The magnitude of adult ODP assessed by single-unit recordings was about one-half of that reported for juveniles (Gordon and Stryker 1996; Fischer et al. 2004; Rao et al. 2004). Moreover, the effect of MD was primarily expressed in deep cortical layers, a later stage of cortical processing compared with more superficial layers.

The size of the deprivation effect that we found in adults is smaller than previously reported for juveniles, but the data are consistent and statistically significant. We have confidence in

A

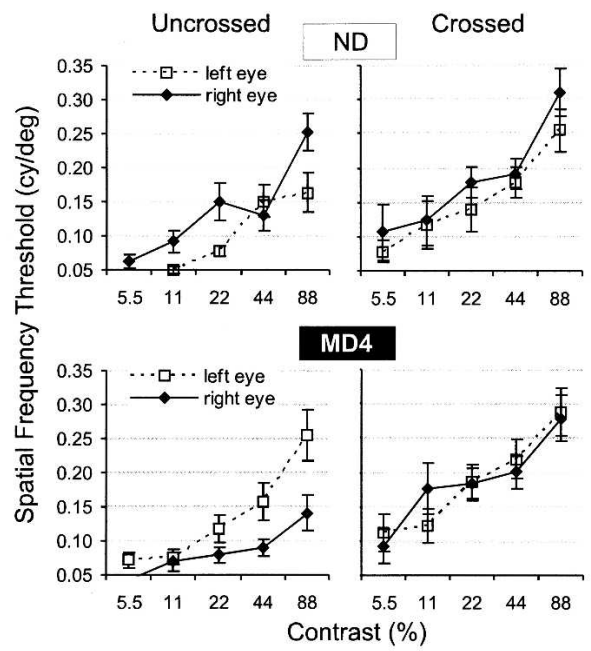

B
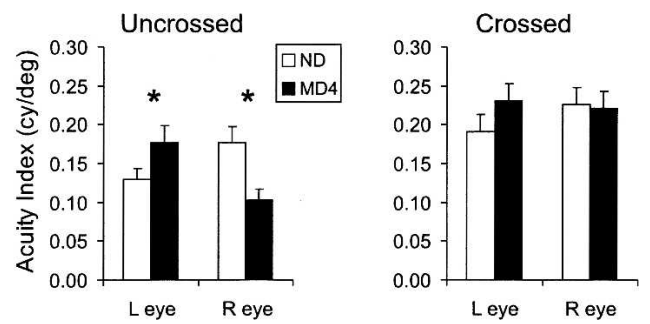

Figure 8. MD alters the visual acuity of the uncrossed (ipsilateral) pathway in adult mice (P90-P390). (A) Spatial frequency threshold (highest spatial frequency that produces a response above criterion) is shown as a function of contrast of the grating stimulus for non-deprived (top) and 4-d right eye MD mice (bottom). Each point is the mean of 11-15 hemispheres. Error bars indicate SEM. (B) Acuity indexes (the average of spatial frequency thresholds at $22 \%, 44 \%$, and $88 \%$ contrast) are shown for the uncrossed and crossed pathways of each eye (each value is the average of 13-15 hemispheres). Asterisk $\left(^{*}\right)$ indicates a significant difference between non-deprived and MD groups $(P \leq 0.03$, Bonferroni post-hoc test). Error bars indicate SEM. 
the validity of our data for the following reasons. First, recordings were performed blind to the deprivation status of the animal. Second, the size of our MD effect (right hemisphere minus left hemisphere $\mathrm{CBI}$ ) was relatively large, about one-half of the effect size previously observed in juveniles (left hemisphere control minus left hemisphere deprived). Note that previous studies recorded only from the left hemisphere. Third, we have a within animal measure of plasticity, which is the left/right hemispheric difference in CBI, whereas previous studies compared deprived and non-deprived animals. A within animal measure is likely to be more sensitive because it eliminates inter-animal variability.

Our single-unit results are important because single-unit recording is the traditional standard for assaying neuronal responses in the visual cortex. In Hubel and Wiesel's seminal single-unit studies (Wiesel and Hubel 1963; Hubel and Wiesel 1970), the critical period in cats was thought to end before three months of age (i.e., pre-pubertal), but this supposition was based on results of only a few animals. Subsequently, the exact temporal span of the critical period was refined in studies that focused more extensively on older animals (Olson and Freeman 1980; Jones et al. 1984). First, ODP in cats was found to extend somewhat past six months of age (Jones et al. 1984). Later, by recording from both hemispheres and tracking the laminar location of recorded units, Daw et al. (1992) found that ODP persists in the extragranular layers as late as 12 mo of age, which is just after puberty, but not at 18 mo of age.

A potentially important factor overlooked in prior singleunit studies is anesthesia. In our previous VEP study, we found that adult ODP could not be observed when the animal's anesthesia contained barbiturate, whereas juvenile ODP was unaffected by barbiturate (Pham et al. 2004). Daw et al. (1992) and Jones et al. (1984) used halothane, which might have contributed to their findings of late plasticity. Another important factor may be cortical depth. We have found that ODP in adult V1 is most prominent in deep layers (approximate layers 5 and 6) and least prominent at depths approximating layer 4 . However, previous studies did not sample deep layers sufficiently. For example, in their study of adult rats, Pizzorusso et al. (2002) appear to have sampled only the upper half of V1 and did not find adult ODP. The only laminar study of ODP in juvenile mice also did not sample deep layers sufficiently, but this study hinted that plasticity was greatest in the deep layers (Gordon and Stryker 1996). A third factor may be bilateral recording. This should enhance detection of adult ODP, because we have found significant plastic changes occur both contralateral and ipsilateral to the deprived eye. Assay procedures not optimized for these factors likely account for past failures to observe ODP in adults.

\section{VEP reveals a robust ODP that extends into middle-age}

To characterize the properties of adult ODP, we used VEP, a rapid and efficient recording method to generate contrast and spatial frequency sensitivity curves. We first asked whether adult ODP disappears after young adulthood, as suggested by Daw et al. (1992). To our knowledge, previous mouse studies included only a few animals past the age of $6 \mathrm{mo}$; the bulk of the data were derived from animals 2-4 mo old, a young age compared to the $>2$-yr lifespan of mice. Here, we have recorded from a group of 20 fully mature mice (average age about nine months) and 10 young adult mice (average about four months). We found no decline of plasticity between young adults and mature adults, and therefore conclude that adult ODP is not a disappearing residual of developmental plasticity.

Another issue concerning adult ODP is its time course. Previous reports have suggested that in adult mice at least four days of $\mathrm{MD}$ are required to produce measurable changes in ocular dominance (Sawtell et al. 2003; Frenkel and Bear 2004; He et al.
2006; Hofer et al. 2006). This differs from juveniles where, in some experiments, MD for as little as two days has been sufficient (Gordon and Stryker 1996; Sawtell et al. 2003). In our present study, an MD effect was observed after only $24 \mathrm{~h}$ of monocular experience. This suggests that adult ODP is rapid in onset, but in its early stages it is not equally detectable by all methods. It is possible that our method is more sensitive because we used a Fourier spectral analysis to identify the visually evoked signal based on the temporal frequency of the stimulus. Another possibility is that our visual stimulus is more optimal. VEP studies from other laboratories have used a highly repetitive stimulus (hundreds of cycles) at very high contrast (>90\%). We have obtained preliminary evidence that these intense stimulation protocols do not reveal adult ODP as effectively as our swept stimulus, possibly because of habituation of high amplitude responses (Q. Fischer and T. Pham, unpubl.). In any case, the very early stages of the plasticity process appear to have an impact on visual cortical function and can be detected using our method of recording swept VEPs.

Finally, we asked whether adult MD alters the sensitivity of the visual system as reflected in spatial frequency thresholds. Human studies have shown that VEP responses can be used to accurately predict perceptual acuity (Tyler et al. 1979; Allen et al. 1986). To estimate acuity, we recorded VEP responses near the threshold of visual detection, using gratings that varied from high spatial frequency to low spatial frequency. This "swept stimulus" technique tends to underestimate acuity slightly (Tyler et al. 1979). Nevertheless, our algorithm to estimate acuity based on the amplitude of the VEP compares reasonably well with results from behavioral studies. We found that the spatial frequency threshold of visual cortex in normal C57Bl/6 mice is 0.30 cycles/degree at $88 \%$ contrast. Using an eye tracking behavioral assay, the acuity of $\mathrm{C} 57 \mathrm{Bl} / 6$ mice was found to be about 0.39 cycles/degree (Prusky et al. 2004; Douglas et al. 2005). Using the behavioral visual water task, the acuity of $\mathrm{C} 57 \mathrm{Bl} / 6$ mice was found to be between 0.38 and 0.5 cycles/degree (Prusky et al. 2000; Wong and Brown 2006).

In the present study, four days of MD changed our index of visual acuity. The effect of MD on the spatial frequency threshold occurred primarily in the uncrossed pathway, which represents the binocular hemifield contralateral to the tested eye. The uncrossed pathway might be more plastic than the crossed pathway because of differences in their anatomy and physiology. In mice, $>95 \%$ of retinal projections are contralateral; $<5 \%$ are ipsilateral. ODP results from the interaction between this major contralateral and minor ipsilateral pathway. If adult ODP results from a competitive process (one pathway gains at the expense of the other), then the ipsilateral pathway will show greater proportional change than the contralateral pathway.

Our electrophysiology data are in agreement with behavioral experiments. Using an eye tracking behavioral assay, Prusky and Douglas found changes in acuity immediately following MD (Douglas et al. 2004). Using a visual water task they did not find evidence of an MD effect in young adults (Prusky and Douglas 2003); however, this result was likely an artifact of the prolonged period of binocular vision required for this form of testing, which allowed the effects of MD to reverse. We found previously that adult ODP is less persistent than juvenile ODP (Pham et al. 2004).

\section{Relation to observations of adult plasticity in primary somatosensory cortex (SI)}

It was thought previously that $\mathrm{S} 1$ had different plastic properties than V1, because map plasticity could be demonstrated in adult S1 (Allard et al. 1991), but not in adult V1. But recent findings of adult ODP suggest that plastic properties of V1 and S1 are con- 
served. There are striking similarities between our current findings and reports of adult plasticity in S1. Similar to our results in V1, S1 "barrel" cortex plasticity was observed in mature adults in the extragranular layers (Fox 1992, 1994; Glazewski and Fox 1996; Skibinska et al. 2000; Wallace et al. 2001). Unfortunately, the majority of electrophysiological studies of S1 have examined only layers 2-4. However, a 2-deoxyglucose labeling study by Skibinska et al. (2000) found that the plasticity in mature mice ( 11 mo of age), compared to that in juvenile mice ( $4 \mathrm{wk}$ of age), was reduced in layers $2 / 3$, absent in layer 4 , but fully retained in layer 5 . Also, some effects of whisker deprivation in adult S1 can be detected after 1-2 d of deprivation (Barth et al. 2000), in agreement with our observation of rapid adult ODP. Finally, adult plasticity in both S1 and V1 is likely to be cortically mediated, because it is disrupted by direct application of muscimol to S1 (Wallace et al. 2001), or targeted genetic deletion of NMDA receptor subunits in V1 (Sawtell et al. 2003).

\section{Concluding remarks}

In contrast to the clear demonstrations of adult ODP in rodents, comparable data for cats and monkeys, the classical experimental animals for visual research, are conspicuously lacking. Whereas the inability to detect adult ODP in cats and monkeys may be related to methodological limitations, it is also possible that adult ODP in rodents is of greater magnitude, or even fundamentally different, than in higher mammals. The lissencephalic rodent cortex is clearly different from the gyrencephalic cortex of carnivores and primates. Despite these differences, however, a VEP study of two human subjects reported rapid adult ODP induced by either monocular occlusion or anisometropia (Tyler and Kaitz 1977). Thus, our findings should act as a stimulus for further work on adult cortical plasticity in higher mammals.

Our findings should not be interpreted to mean that there is no critical period for ODP. There are well-established developmental changes in the properties of ODP, which may be driven by the down-regulation of cAMP/CRE-mediated gene expression (Pham et al. 1999) and/or the upregulation of extracellular matrix proteins and myelin (Pizzorusso et al. 2002; McGee et al. 2005). Adult ODP is masked by barbiturate anesthesia and is less persistent than developmental ODP (Pham et al. 2004). The challenge before us is to understand how the plastic mechanisms found in adult V1 differ from those present in development, which may yield insights into how impaired vision in adults can be permanently improved.

\section{Materials and Methods}

All procedures used in this study were approved by the Institutional Animal Care and Use Committees of Baylor College of Medicine or the University of Oregon and conform to the guidelines of the National Institutes of Health and the Society for Neuroscience.

\section{Mice}

All mice used in this study were of the C57BL/6 strain. Mice were obtained from Jackson Laboratory (Bar Harbor, $\mathrm{ME}$ ), Simonsen Laboratories (Gilroy, CA), Charles River (Wilmington, MA) or Taconic Farms (Germantown, NY), or bred directly from these stocks. Mice were housed under a standard 12-h light/12-h dark schedule, unless otherwise noted.

\section{Single-unit recordings}

MD, accomplished by eyelid suture of either the right or left eye, was performed under isoflurane anesthesia (Attane 1\%-2\%, Minrad). Mice were checked daily to ensure that the lids remained closed and that there was no infection. MD lasted either four days (four mice) or nine days (two mice). Single-unit recordings were made in five non-deprived mice (P55-P110) and six MD mice (P60-P106) using the procedures of Fischer et al. (2004), with two important exceptions. First, we used urethane anesthesia $(1.5 \mathrm{mg} / \mathrm{g}$ body weight, i.p.). Second, we recorded bilaterally (alternating between hemispheres) from V1b in both the left and right hemispheres of non-deprived mice or the hemisphere contralateral and ipsilateral to the deprived eye of MD mice. All recordings were made blind to the deprivation condition of the animals (non-deprived, right eye MD, or left eye MD). Briefly, we recorded five to seven cells (at 100 - $\mu$ m intervals) in each of three vertical penetrations spaced evenly $(\sim 250-\mu \mathrm{m}$ intervals) across the mediolateral extent of V1b to avoid sampling bias. Care was taken to ensure that receptive field topography was appropriate for V1b. That is, as the electrode was moved from medial to lateral ( 2.7 to $3.3 \mathrm{~mm}$ lateral to midline), receptive fields moved centrally (from $25^{\circ}$ to $0^{\circ}$ ) within the contralateral hemifield (Drager 1978; Wagor et al. 1980; Gordon and Stryker 1996).

Receptive fields of isolated single-units were plotted on a tangent screen, with the vertical meridian defined as the animal's midline. The preferred direction, orientation, size, and velocity of the stimulus were determined. Ocular dominance was then assigned using a seven-point scale (one contralateral eye only, four both eyes equally, seven ipsilateral eyes only). Ocular dominance histograms were constructed for each hemisphere for each group of mice. For each hemisphere (or depth) in each animal or group of animals we also calculated a contralateral bias index (CBI) using the formula: CBI $=\left[\left(\mathrm{n}_{1}-\mathrm{n}_{7}\right)+(2 / 3)\left(\mathrm{n}_{2}-\mathrm{n}_{6}\right)+(1 /\right.$ $\left.3)\left(n_{3}-n_{5}\right)+N\right] / 2 N$, were $N$ is the total number of cells and $n_{i}$ is the number of cells in OD category i. A CBI of 0 would indicate all cells were driven exclusively by the ipsilateral eye, while a CBI
L eye, L hem
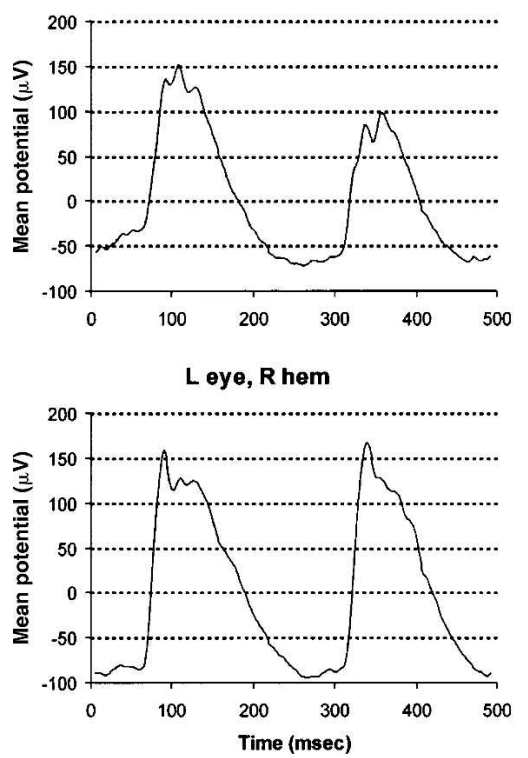

Figure 9. VEP waveforms in a monocularly deprived mouse. VEPs were recorded from the right and left hemisphere of a mouse following monocular deprivation of the right eye. The visual stimulus was a reversal grating presented at a fixed contrast of $80 \%$ for a duration of $10 \mathrm{sec}$. The grating reversed contrast at a frequency of $2 \mathrm{~Hz}$. The stimulus was presented alternately to the right and left eye for a total of four trials each. $L$ hem, left hemisphere; $R$ hem, right hemisphere; $L$ eye, left eye; $R$ eye, right eye.

\section{Learning \& Memory} www.learnmem.org 
of 1 would indicate all cells were driven by the contralateral eye. The CBI of non-deprived mice is $\sim 0.671$, that is biased toward the contralateral eye. Responses for each cell were also rated on a three-point scale for both the strength of the visual response and spontaneous activity according to the methods of Fischer et al. (2004). Following completion of the third (final) penetration in each hemisphere a pair of electrolytic lesions was made to mark recording depth. Animals were deeply anesthetized with Nembutal $(100 \mathrm{mg} / \mathrm{kg}$, i.p.; Abbott Labs) and perfused with $4 \%$ paraformaldehyde in phosphate buffer. The brain was removed, postfixed, sectioned at $50 \mu \mathrm{m}$, and stained with Bisbenzimide (Sigma) for the reconstruction of electrode tracts.

\section{VEP recordings}

VEPs were recorded from 61 non-deprived and MD mice aged P90-P390, according to the methods of Pham et al. (2004) and Lickey et al. (2004). We have already published an example of the VEP waveforms for a non-deprived adult (Fig. 2; Lickey et al. 2004). Here, for comparison, we provide an example of the VEP waveforms for an adult subject to MD (Fig. 9). In all cases MD was of the right eye, and lasted for a period of $4 \mathrm{~d}$ unless otherwise noted. PowerDiva software from Anthony Norcia (Smith Kettlewell Institute of Visual Sciences, San Francisco, CA) was used for data acquisition and analysis. The visual stimulus was a horizontal sinusoidal grating covering the central $90^{\circ}$ of the visual field $\left(45^{\circ}\right.$ on either side of the midline). The grating reversed phase 6.2 times per second $(\sim 3 \mathrm{~Hz})$. One stimulus presentation (one trial) consisted of a spatial frequency sweep decreasing from 0.65 to 0.05 cycles/degree in 10 log steps. Trials were repeated at each of five different contrasts $(5.5 \%, 11 \%, 22 \%, 44 \%$, and $88 \%)$. Trials alternated between right and left eyes until 16 trials were completed for each combination of spatial frequency and contrast.

\section{Analysis of VEP data}

The response was extracted from noise using digital filtration (Tang and Norcia 1995). The visually evoked response has frequencies that are even multiples (harmonics) of the stimulus frequency. We defined the VEP amplitude as the sum of the amplitudes for the second and fourth harmonics (Lickey et al. 2004; Pham et al. 2004). Since the stimulus frequency was $3 \mathrm{~Hz}$, the signal amplitude was the sum of amplitudes at 6 and $12 \mathrm{~Hz}$. Relative (normalized) amplitude was defined as the amplitude of the VEP divided by the mean of all VEP amplitudes for contrasts $\geq 22 \%$ and spatial frequencies $\geq 0.14$ cycles/degree recorded in the same hemisphere. A noise component was calculated for each component of the signal. The noise component was the mean amplitude at two frequencies straddling the signal frequency; e.g., for the second harmonic (6 Hz component), the noise was the average of the two amplitudes at 5 and $7 \mathrm{~Hz}$; for the fourth harmonic (12 Hz component), the noise was the average amplitude at 11 and $13 \mathrm{~Hz}$. The sum of the two noise components is the noise that is plotted in Figure 4 (bottom). Data from a hemisphere were rejected if the maximum VEP amplitude was $\leq$ three times the average noise in that hemisphere.

Spatial frequency thresholds were derived using the following algorithm. The data of one spatial frequency sweep fell into 11 bins of decreasing spatial frequency. In the first bin the spatial frequency was 0.65 cycles/degree, in the last bin it was 0.05 cycles/degree. The mean and standard deviation of noise was calculated across the 11 bins. The signal in each bin was scored as a hit if the signal amplitude exceeded the mean plus one standard deviation of the noise. If two consecutive bins failed to score a hit, the second bin of the pair was scored a failure. The threshold was then defined as the spatial frequency of the first hit after the last failure. If there were no hits, the threshold was arbitrarily assigned the value of 0.038 cycles/degree. If there were no failures, the threshold was assigned the value of 0.65 .

For each hemisphere of each animal or group of animals we also calculated an ocular dominance index (ODI) according to the formula: $\mathrm{ODI}=\mathrm{X} /(\mathrm{X}+\mathrm{U})$, where $\mathrm{X}=$ the VEP amplitude of the crossed pathway and $U=$ the VEP amplitude of the uncrossed pathway. ODIs were calculated using only the data obtained at $88 \%$ contrast and a spatial frequency of 0.05 cycles/degree.

\section{Acknowledgments}

This research was supported by National Eye Institute grant RO1 EY014238, National Institute of Mental Health grant KO8 MH01971, and a start up grant from Baylor College of Medicine to T.A.P. We thank Barbara Gordon-Lickey and Nigel Daw for help with data analysis and critical reading of the manuscript. We thank Anthony Norcia for his generous donation of data collection and analysis software. Finally, we thank Seigo Suzuki for assistance with surgical procedures and Jim Legare for animal care.

\section{References}

Allard, T., Clark, S.A., Jenkins, W.M., and Merzenich, M.M. 1991. Reorganization of somatosensory area $3 \mathrm{~b}$ representations in adult owl monkeys after digital syndactyly. J. Neurophysiol. 66: 1048-1058.

Allen, D., Norcia, A.M., and Tyler, C.W. 1986. Comparative study of electrophysiological and psychophysical measurement of the contrast sensitivity function in humans. Am. J. Optom. Physiol. Opt. 63: $442-449$.

Barth, A.L., McKenna, M., Glazewski, S., Hill, P., Impey, S., Storm, D., and Fox, K. 2000. Upregulation of cAMP response element-mediated gene expression during experience-dependent plasticity in adult neocortex. J. Neurosci. 20: 4206-4216.

Daw, N.W., Fox, K., Sato, H., and Czepita, D. 1992. Critical period for monocular deprivation in the cat visual cortex. J. Neurophysiol. 67: 197-202.

Douglas, R.M., Alam, N.M., and Prusky, G.T. 2004. Enhancement of spatial vision in adult mice induced by monocular deprivation. In Abstract Viewer/Itinerary Planner, Program no. 866.15. Society for Neuroscience, Washington, DC.

Douglas, R.M., Alam, N.M., Silver, B.D., McGill, T.J., Tschetter, W.W., and Prusky, G.T. 2005. Independent visual threshold measurements in the two eyes of freely moving rats and mice using a virtual-reality optokinetic system. Vis. Neurosci. 22: 677-684.

Drager, U.C. 1975. Receptive fields of single cells and topography in mouse visual cortex. J. Comp. Neurol. 160: 269-286.

Drager, U.C. 1978. Observations on monocular deprivation in mice. $J$. Neurophysiol. 41: 28-42.

Fagiolini, M., Pizzorusso, T., Berardi, N., Domenici, L., and Maffei, L. 1994. Functional postnatal development of the rat primary visual cortex and the role of visual experience: Dark rearing and monocular deprivation. Vision Res. 34: 709-720.

Fischer, Q.S., Beaver, C.J., Yang, Y., Rao, Y., Jakobsdottir, K.B., Storm, D.R., McKnight, G.S., and Daw, N.W. 2004. Requirement for the RIIbeta isoform of PKA, but not calcium-stimulated adenylyl cyclase, in visual cortical plasticity. J. Neurosci. 24: 9049-9058.

Fox, K. 1992. A critical period for experience-dependent synaptic plasticity in rat barrel cortex. J. Neurosci. 12: 1826-1838.

Fox, K. 1994. The cortical component of experience-dependent synaptic plasticity in the rat barrel cortex. J. Neurosci. 14: 7665-7679.

Frenkel, M.Y. and Bear, M.F. 2004. How monocular deprivation shifts ocular dominance in visual cortex of young mice. Neuron 44: 917-923.

Glazewski, S. and Fox, K. 1996. Time course of experience-dependent synaptic potentiation and depression in barrel cortex of adolescent rats. J. Neurophysiol. 75: 1714-1729.

Gordon, J.A. and Stryker, M.P. 1996. Experience-dependent plasticity of binocular responses in the primary visual cortex of the mouse. $J$. Neurosci. 16: 3274-3286.

Guire, E.S., Lickey, M.E., and Gordon, B. 1999. Critical period for the monocular deprivation effect in rats: Assessment with sweep visually evoked potentials. J. Neurophysiol. 81: 121-128.

He, H.Y., Hodos, W., and Quinlan, E.M. 2006. Visual deprivation reactivates rapid ocular dominance plasticity in adult visual cortex. $J$. Neurosci. 26: 2951-2955.

Hensch, T.K., Fagiolini, M., Mataga, N., Stryker, M.P., Baekkeskov, S., and Kash, S.F. 1998. Local GABA circuit control of experience-dependent plasticity in developing visual cortex. Science 282: $1504-1508$.

Hofer, S.B., Mrsic-Flogel, T.D., Bonhoeffer, T., and Hubener, M. 2006. Prior experience enhances plasticity in adult visual cortex. Nat. Neurosci. 9: 127-132.

Hubel, D.H. and Wiesel, T.N. 1962. Receptive fields, binocular interaction and functional architecture in the cat's visual cortex. J. Physiol. 160: 106-154.

Hubel, D.H. and Wiesel, T.N. 1970. The period of susceptibility to the 
physiological effects of unilateral eye closure in kittens. J. Physiol. 206: $419-436$.

Issa, N.P., Trachtenberg, J.T., Chapman, B., Zahs, K.R., and Stryker, M.P. 1999. The critical period for ocular dominance plasticity in the Ferret's visual cortex. J. Neurosci. 19: 6965-6978.

Jones, K.R., Spear, P.D., and Tong, L. 1984. Critical periods for effects of monocular deprivation: Differences between striate and extrastriate cortex. J. Neurosci. 4: 2543-2552.

LeVay, S., Wiesel, T.N., and Hubel, D.H. 1980. The development of ocular dominance columns in normal and visually deprived monkeys. J. Comp. Neurol. 191: 1-51.

Lickey, M.E., Pham, T.A., and Gordon, B. 2004. Swept contrast visual evoked potentials and their plasticity following monocular deprivation in mice. Vision Res. 44: 3381-3387.

McGee, A.W., Yang, Y., Fischer, Q.S., Daw, N.W., and Strittmatter, S.M. 2005. Experience-driven plasticity of visual cortex limited by myelin and Nogo receptor. Science 309: 2222-2226.

Olson, C.R. and Freeman, R.D. 1980. Profile of the sensitive period for monocular deprivation in kittens. Exp. Brain Res. 39: 17-21.

Pham, T.A., Impey, S., Storm, D.R., and Stryker, M.P. 1999. CRE-mediated gene transcription in neocortical neuronal plasticity during the developmental critical period. Neuron 22: 63-72.

Pham, T.A., Graham, S.J., Suzuki, S., Barco, A., Kandel, E.R., Gordon, B., and Lickey, M.E. 2004. A semi-persistent adult ocular dominance plasticity in visual cortex is stabilized by activated CREB. Learn. Mem. 11: 738-747.

Pizzorusso, T., Medini, P., Berardi, N., Chierzi, S., Fawcett, J.W., and Maffei, L. 2002. Reactivation of ocular dominance plasticity in the adult visual cortex. Science 298: 1248-1251.

Prusky, G.T. and Douglas, R.M. 2003. Developmental plasticity of mouse visual acuity. Eur. J. Neurosci. 17: 167-173.

Prusky, G.T., West, P.W., and Douglas, R.M. 2000. Behavioral assessment of visual acuity in mice and rats. Vision Res. 40: 2201-2209.

Prusky, G.T., Alam, N.M., Beekman, S., and Douglas, R.M. 2004. Rapid quantification of adult and developing mouse spatial vision using a virtual optomotor system. Invest. Ophthalmol. Vis. Sci. 45: 4611-4616.

Rao, Y., Fischer, Q.S., Yang, Y., McKnight, G.S., LaRue, A., and Daw, N.W. 2004. Reduced ocular dominance plasticity and long-term potentiation in the developing visual cortex of protein kinase A RII $\alpha$ mutant mice. Eur. J. Neurosci. 20: 837-842.

Sawtell, N.B., Frenkel, M.Y., Philpot, B.D., Nakazawa, K., Tonegawa, S. and Bear, M.F. 2003. NMDA receptor-dependent ocular dominance plasticity in adult visual cortex. Neuron 38: 977-985.

Shatz, C.J. and Stryker, M.P. 1978. Ocular dominance in layer IV of the cat's visual cortex and the effects of monocular deprivation. $J$. Physiol. 281: 267-283.

Skibinska, A., Glazewski, S., Fox, K., and Kossut, M. 2000. Age-dependent response of the mouse barrel cortex to sensory deprivation: A 2-deoxyglucose study. Exp. Brain Res. 132: 134-138.

Tagawa, Y., Kanold, P.O., Majdan, M., and Shatz, C.J. 2005. Multiple periods of functional ocular dominance plasticity in mouse visual cortex. Nat. Neurosci. 8: 380-388.

Tang, Y. and Norcia, A.M. 1995. An adaptive filter for steady-state evoked responses. Electroencephalogr. Clin. Neurophysiol. 96: 268-277.

Tyler, C.W. and Kaitz, M.F. 1977. Binocular interactions in the human visual evoked potential after short-term occlusion and anisometropia. Invest. Ophthalmol. Vis. Sci. 16: 1070-1076.

Tyler, C.W., Apkarian, P., Levi, D.M., and Nakayama, K. 1979. Rapid assessment of visual function: An electronic sweep technique for the pattern visual evoked potential. Invest. Ophthalmol. Vis. Sci. 18: 703-713.

Wagor, E., Mangini, N.J., and Pearlman, A.L. 1980. Retinotopic organization of striate and extrastriate visual cortex in the mouse. $J$. Comp. Neurol. 193: 187-202.

Wallace, H., Glazewski, S., Liming, K., and Fox, K. 2001. The role of cortical activity in experience-dependent potentiation and depression of sensory responses in rat barrel cortex. J. Neurosci. 21: $3881-3894$.

Wiesel, T.N. and Hubel, D.H. 1963. Single-cell responses in striate cortex of kittens deprived of vision in one eye. J. Neurophysiol. 26: $1003-1017$

Wong, A.A. and Brown, R.E. 2006. Visual detection, pattern discrimination and visual acuity in 14 strains of mice. Genes Brain Behav. 5: 389-403.

Received August 1, 2006; accepted in revised form February 20, 2007.

\section{Learning \& Memory}




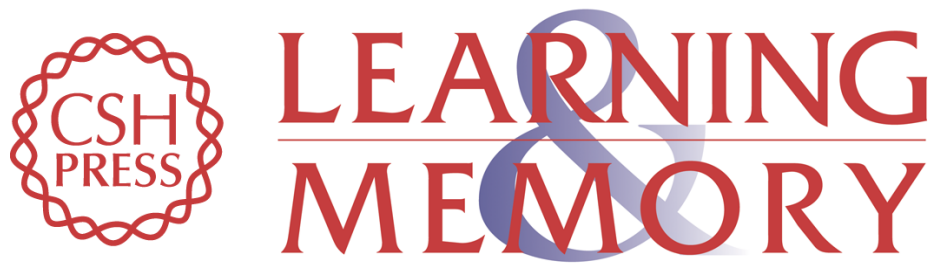

\section{Monocular deprivation in adult mice alters visual acuity and single-unit activity}

Quentin S. Fischer, Aundrea Graves, Scott Evans, et al.

Learn. Mem. 2007, 14:

Access the most recent version at doi:10.1101//m.392107

References This article cites 44 articles, 16 of which can be accessed free at:

http://learnmem.cshlp.org/content/14/4/277.full.html\#ref-list-1

License

Email Alerting Receive free email alerts when new articles cite this article - sign up in the box at the Service top right corner of the article or click here. 